\title{
Influence of ethnicity on the distribution of genetic polymorphisms associated with risk of chronic liver disease in South American populations
}

Ana Cecilia Pontoriero ${ }^{1}$, Julieta Trinks ${ }^{1,2^{*}}$, María Laura Hulaniuk' ${ }^{1}$ Mariela Caputo ${ }^{2,3}$, Lisandro Fortuny ${ }^{4}$, Leandro Burgos Pratx ${ }^{4}$, Analía Frías ${ }^{5}$, Oscar Torres $^{5}$, Félix Nuñez $^{4}$, Adrián Gadano $^{6}$, Pablo Argibay ${ }^{1,2^{\wedge}}$, Daniel Corach ${ }^{2,3}$ and Diego Flichman $n^{2,7}$

\begin{abstract}
Background: The global burden of chronic liver disease is rising. Besides environmental, behavioral, viral and metabolic factors, genetic polymorphisms in patatin-like phospholipase-3 (PNPLA3) and vitamin D receptor (VDR) genes have been related to the development of chronic liver disease and progression towards liver cancer. Although their prevalence differs remarkably among ethnic groups, the frequency of these polymorphisms in South American populations -whose genetic background is highly admixed- has been poorly studied. Hence, the aim of this study was to characterize polymorphisms related to chronic liver disease and their association with the genetic ancestry of South American populations.

Results: DNA samples from 258 healthy unrelated male volunteers were analyzed. The frequencies of $\mathrm{G}$ and $\mathrm{C}$ alleles of rs738409 polymorphism (PNPLA3 gene) were $74 \%$ and $26 \%$, respectively; whereas the bAt (CCA) haplotype (VDR gene) was observed in $32.5 \%$ of the samples. The GG genotype of PNPLA3 rs738409 and the bAt (CCA) haplotype -associated with an increased risk of chronic liver disease and progression towards liver cancer- were significantly more frequent among samples exhibiting maternal and paternal Native American haplogroups (63.7 \% and $64.6 \%)$, intermediate among admixed samples (45.1\% and $44.9 \% ; p=0.03)$ and the lowest for Non-native American ancestry (30.1\% and $29.6 \% ; p=0.001$ and $p=0.0008)$.

Conclusions: These results suggest that individuals with Native American ancestry might have a high risk of chronic liver disorders and cancer. Furthermore, these data not only support the molecular evaluation of ancestry in multi-ethnic population studies, but also suggest that the characterization of these variants in South American populations may be useful for establishing public health policies aimed at high risk ethnic communities.
\end{abstract}

Keywords: Chronic liver disease, Liver cancer, PNPLA3 gene, VDR gene, Polymorphism, Ethnicity, South America

\footnotetext{
* Correspondence: julieta.trinks@hospitalitaliano.org.ar

Deceased

'Instituto de Ciencias Básicas y Medicina Experimental (ICBME), Hospital

Italiano de Buenos Aires, Potosí 4240, C1199ACL Buenos Aires, Argentina

${ }^{2}$ National Scientific and Technical Research Council (CONICET), Av. Rivadavia

1917, C1033AAJ Buenos Aires, Argentina

Full list of author information is available at the end of the article
} 


\section{Background}

The global health and economic burden of chronic liver disease is substantial and on the rise, due in part to the growing incidence of hepatic steatosis and cirrhosis [1, 2]. The increase in cirrhosis, along with a surge in the prevalence of its risk factors such as alcohol abuse and infection with hepatitis B virus (HBV) and/or hepatitis C virus $(\mathrm{HCV})$, has resulted in a growing incidence of primary liver cancer [3]. The major histological subtype of primary liver malignancies, hepatocellular carcinoma (HCC), represents the end stage of the natural history of chronic liver disease and has become the third leading cause of cancer-related mortality worldwide and the major indication for liver transplant in Europe and USA [4].

Hepatic steatosis is the most common pathological finding in liver biopsies around the world [5]. Although its association with liver inflammation is an evidence suggestive of alcohol-induced liver injury, it has also been described in individuals with no significant history of alcohol ingestion [6,7]. The term non-alcoholic fatty liver disease (NAFLD) has been adopted to cover the full spectrum of metabolic fatty liver disorders, as it ranges from simple steatosis to non-alcoholic steatohepatitis (NASH) and fibrosis with severe disruption of the normal architecture and function of the liver leading to cirrhosis [6, 7]. Increasing rates of NAFLD and $\mathrm{NASH}$-the most common forms of chronic liver disease in Western countries- have been reported as the worldwide prevalence of obesity continues to rise [5-7]. In turn, obesity, NAFLD and NASH are increasingly recognized triggers involved in the development of both cirrhosis and HCC [8].

There is consistent evidence that the burden of chronic liver disease is greater among certain ethnic groups, particularly in areas characterized by high rates of chronic infection with hepatitis viruses [9]. Moreover, racial disparities in the prevalence and clinical presentation of chronic liver disorders have frequently been reported. In fact, Hispanics have a higher prevalence of steatohepatitis, NAFLD and cirrhosis, whereas African-Americans are less prone to develop liver failure [10-12].

Besides viral, environmental, behavioral, and metabolic features, genetic factors have also been implicated in the different susceptibility to develop HCC [13]. In the last few years, several studies have established the contribution of single nucleotide polymorphisms (SNPs) in different genes -such as the patatin-like phospholipase-3 (PNPLA3), vitamin D receptor $(V D R)$, transmembrane 6 superfamily member 2 (TM6SF2), beta-parvin (PARVB) and sorting and assembly machinery component 50 (SAMM50) genes- as causal variants for chronic liver diseases and their progression towards HCC [14-17]. This study will focus only on the PNPLA3 and VDR polymorphisms.
A genome-wide association study in a population comprising Hispanic, African-American and Caucasian subjects revealed that the SNP rs738409C $>\mathrm{G}$, which encodes an isoleucine-to-methionine substitution at position 148 (I148M) in the PNPLA3 gene, is the strongest determinant of hepatic steatosis [18]. Many other studies in Asian, European and Latin American populations have since confirmed this association and shown the implication of this genetic variant in chronic liver disorders ranging from NAFLD and NASH to cirrhosis and HCC [19-22].

Moreover, the progression of chronic liver diseases is related to the presence of genetic polymorphisms in the VDR gene. In fact, decreased vitamin D levels have increasingly been recognized in various forms of chronic liver disease and associated with advanced fibrosis [23, 24]. Patients with NAFLD were found to present with reduced vitamin D levels, which were closely related to the histological severity of hepatic steatosis, necroinflammation and fibrosis $[25,26]$.

Several polymorphic sites -such as the bAt (CCA) haplotype consisting of the combination of SNPs rs1544410C (formerly BsmI), rs7975232C (ApaI) and rs731236A (TaqI)have been described in the VDR gene [27]. These combined variants showed a significant association with the fibrosis progression rate and the occurrence of cirrhosis and HCC in Caucasian and Asian patients diagnosed with alcoholinduced liver injury as well as in those chronically infected with HCV [28-30].

The prevalence of these polymorphisms differs among ethnic groups $[18,27]$. However, there is a paucity of information about South American populations, whose genetic background is highly admixed as a result of generations of intermixing between various groups, including Native Americans, Spanish conquistadores, Africans and a large European immigrant population that arrived between 1870 and 1950. In the Argentinean population, for example, further sources of admixture have been introduced by local migration from the rural areas to the cities between 1930 and 1980, as well as by immigration from other South American countries [31, 32]. Hence, the aim of this study was to determine the prevalence of these genetic polymorphisms and their association with the genetic ancestry of South American populations, which may provide useful knowledge for establishing public health policies aimed at high risk ethnic populations.

\section{Results}

The SNPs in PNPLA3 and VDR genes, associated to the development of chronic liver disease and progression towards liver cancer, have been characterized in 258 healthy unrelated male volunteers. The allele and genotype frequencies of these SNPs are presented in Table 1. No significant differences in age were observed among 
Table 1 Allele and genotype frequencies of SNPs rs738409 (PNPLA3), rs1544410, rs7975232, rs731236 and bAt (CCA) haplotype (VDR)

\begin{tabular}{lllll}
\hline & \multicolumn{2}{l}{ Allele frequency } & \multicolumn{2}{l}{ Genotype frequency (\%) } \\
\hline PNPLA3 & & & & \\
rs738409 & G: & 0.74 & GG: & 55.4 \\
& C: & 0.26 & GC/CC: & 44.6 \\
VDR & & & & \\
rs1544410 (Bsml) & C: & 0.78 & CC: & 58.9 \\
& T: & 0.22 & CT/TT: & 41.1 \\
rs7975232 (Apal) & C: & 0.70 & CC: & 50.4 \\
& A: & 0.30 & CA/AA: & 49.6 \\
rs731236 (Taql) & A: & 0.75 & AA: & 55.8 \\
& G: & 0.25 & AG/GG: & 44.2 \\
Haplotype & & & bAt (CCA): & 32.5 \\
& & & Others haplotypes: & 67.5 \\
\hline
\end{tabular}

the individuals carrying the different alleles. The distribution of homozygous and heterozygous carriers was consistent with the expectations of the Hardy-Weinberg equilibrium (chi-square goodness-of-fit test: $p>0.05$ for all SNPs in all groups).

The molecular evaluation of maternal ancestry reveals that $74.4 \%$ of the 258 individuals exhibited Native American lineages (haplogroups A2, B2, C and D1; Fig. 1a); whereas, as regards paternal ancestry, only $23.6 \%$ of the samples showed the Native American Q1a3a haplogroup (Fig. 1b). Moreover, the combined analysis of mitochondrial DNA (mtDNA) and Y-chromosome single-nucleotide polymorphisms (Y-SNPs) in each of the 258 recruited individuals revealed that more than half of them (57.8\%) exhibited maternal Native American haplogroups and paternal Non-native American ancestry (admixed samples); whereas $17.8 \%$ and $24.4 \%$ of the studied samples showed Native American and Non-native American ancestry, respectively.

The four studied SNPs were unevenly distributed among individuals with Non-native American ancestry compared with those with Native American ancestry (maternal or paternal lineages). In fact, the frequencies of bAt (CCA) haplotype (VDR gene) and the GG genotype of rs738409 polymorphism (PNPLA3 gene) were significantly higher among samples with Native American maternal ancestry (63.4\% and $65.4 \%$, respectively) compared with those with Non-native American maternal haplogroups (32.2\% and $38.4 \%$, respectively) ( $p=0.0005$ and $p=0.0008$, respectively; Fig. 2). In addition, these frequencies were unevenly distributed among individuals with Native American paternal ancestry compared with those with Non-native American paternal ancestry, being significantly higher in those with Native American haplogroups (54.9\% and $53.1 \%$, respectively) compared with those with Non-native American haplogroups $(39.8 \%$ and $30.9 \%$,

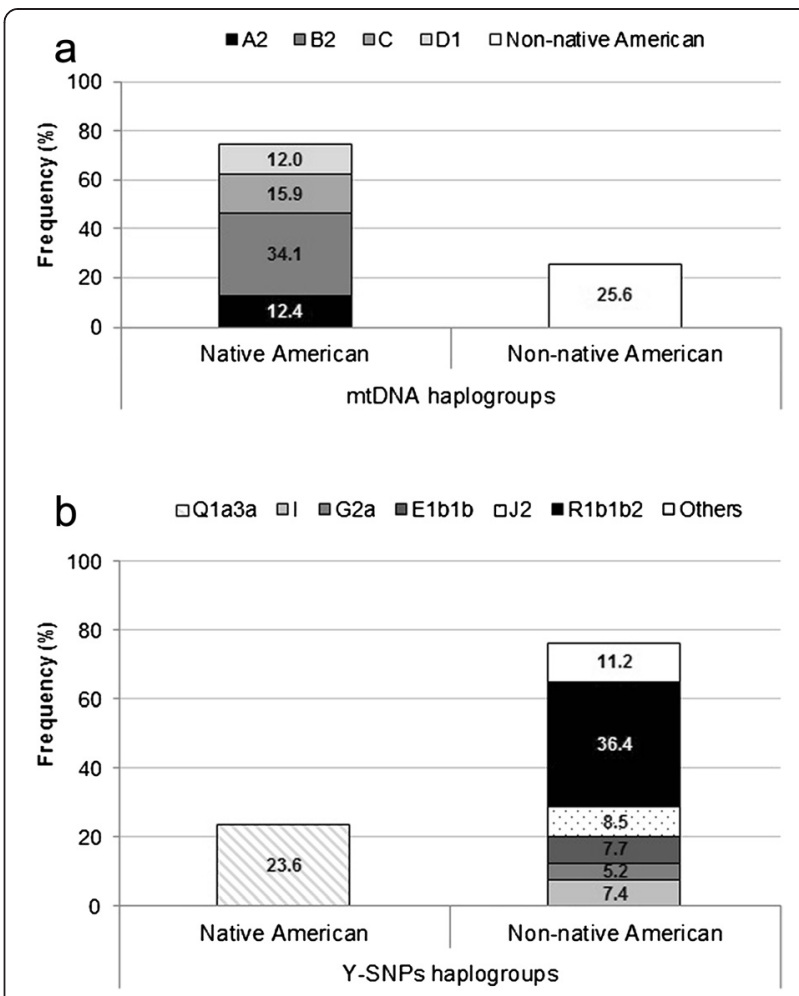

Fig. 1 Genetic ancestry of the study population. Prevalence of (a) mitochondrial DNA (mtDNA) haplogroups and (b) Y-chromosome single-nucleotide polymorphisms (Y-SNPs) among the studied population

respectively) ( $p=0.0424$ and $p=0.0388$, respectively; Fig. 3). Similar results were observed when examining the relationship between polymorphisms in VDR gene and ancestry (Additional files 1 and 2).

When the prevalence of the SNP rs738409 (PNPLA3) was determined according to the combined ancestry of the samples (maternal and paternal lineages), the unfavorable genotype GG was significantly higher among samples with Native American lineages (63.7 \%), intermediate among admixed samples (45.1\%, $p=0.03)$ and the lowest for samples with Non-native American ancestry $(30.1 \%, p=0.001$; Fig. 4$)$.

Similar results were found when the three polymorphisms in VDR gene were examined (Additional file 3). As a consequence, the frequency of the bAt (CCA) haplotype -which is a result of the combination of the three SNPs- was significantly higher among samples with maternal and paternal Native American haplogroups (64.6\%), intermediate among admixed samples (44.9\%, $p=0.03$ ) and the lowest for samples with Non-native American ancestry (29.6\%, $p=0.0008$; Fig. 4).

\section{Discussion}

Chronic liver disease is an important cause of morbidity and mortality around the world. The ethnic disparities 


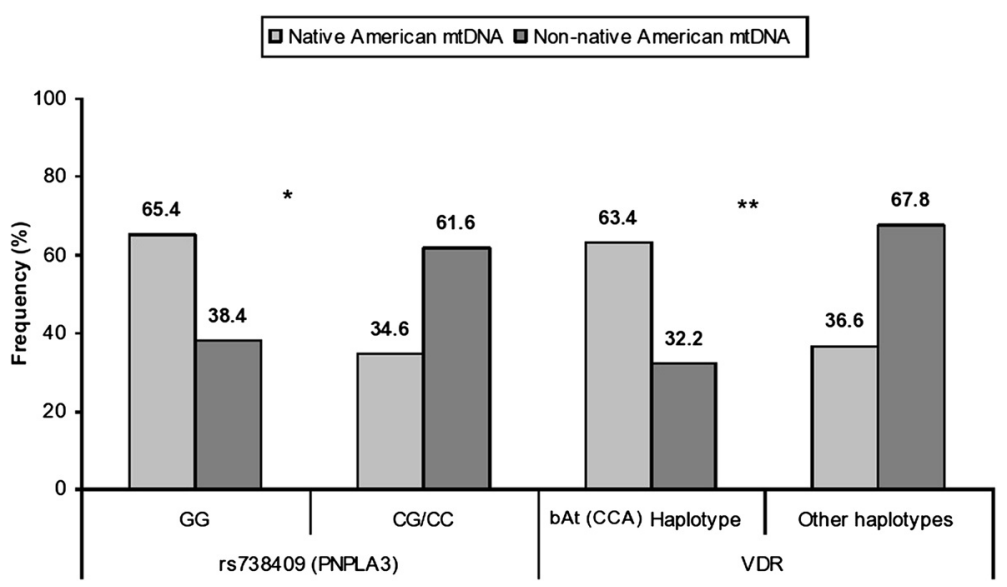

Fig. 2 Influence of maternal ethnic components on the genotype distribution. Prevalence of SNP rs738409 (PNPLA3 gene) and bAt (CCA) haplotype (VDR gene) among samples of Native American and Non-native American maternal ancestry $\left({ }^{*} p=0.0008,{ }^{* *} p=0.0005\right.$ )

reported in its burden, prevalence and clinical presentation are likely attributed to the interaction between environmental, behavioral, and genetic factors $[1,9]$. To the best of our knowledge, this study is the first to analyze ethnicity and polymorphisms associated with susceptibility to chronic liver disease and progression towards HCC in South American populations.

The results presented herein reveal that the distribution of polymorphisms related to the development of chronic liver disease and progression towards liver cancer exhibits statistical significant differences according to ethnicity. In fact, the prevalence of host genetic factors associated with risk of liver steatosis, fibrosis and cirrhosis is significantly lower in individuals with Non-native American ancestry.

In addition to ethnicity, other risk factors have been associated with the development of chronic liver disease, such as older age, male gender, alcohol abuse and HBV and/or HCV infection [1]. To ensure a representative sample of the general population in the region, 258 unrelated male volunteers were enrolled by two blood banks in Buenos Aires city. The exclusion of HBV and/or HCV infected subjects did not affect sample's representativeness as prevalence of $\mathrm{HBV}$ and $\mathrm{HCV}$ infection in Argentina is lower than $1 \%$ [33].

Moreover, the proportion of each ancestry pattern in the collected sample was consistent with previously reported frequencies in the general population [32], and therefore, no ethnic group was over or underrepresented in the sample. In this area of Argentina, the more prevalent ancestry components for maternal and paternal lineages are the European and the Native American, followed -to a lesser extent- by African haplogroups [32]. Therefore, these data suggest that the samples with Non-native American maternal and/or paternal haplogroups analyzed in this study would predominantly exhibit the

$\square$ Native American Y-SNPS $\square$ Non-native American Y-SNPS

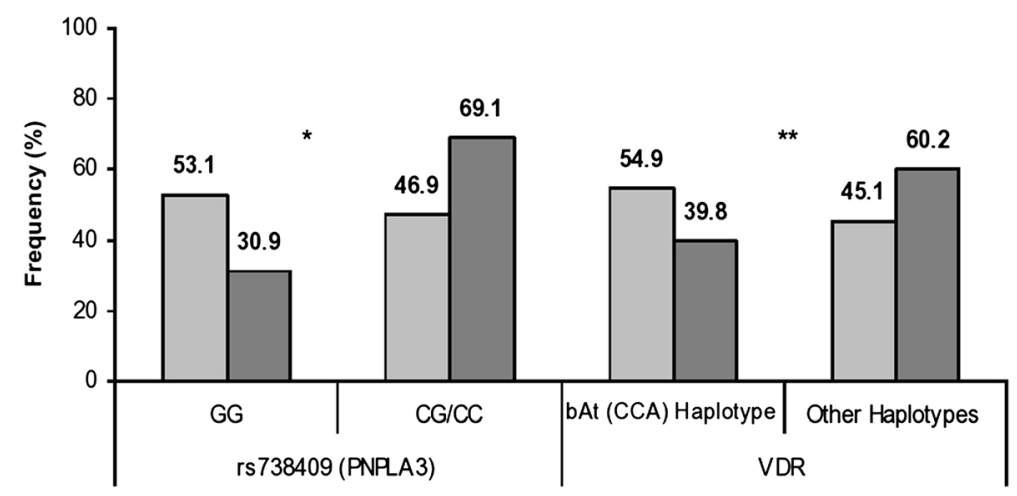

Fig. 3 Influence of paternal ethnic components on the genotype distribution. Prevalence of SNP rs738409 (PNPLA3 gene) and bAt (CCA) haplotype (VDR gene) among samples of Native American and Non-native American paternal ancestry $\left({ }^{*} p=0.0388,{ }^{* *} p=0.0424\right)$ 

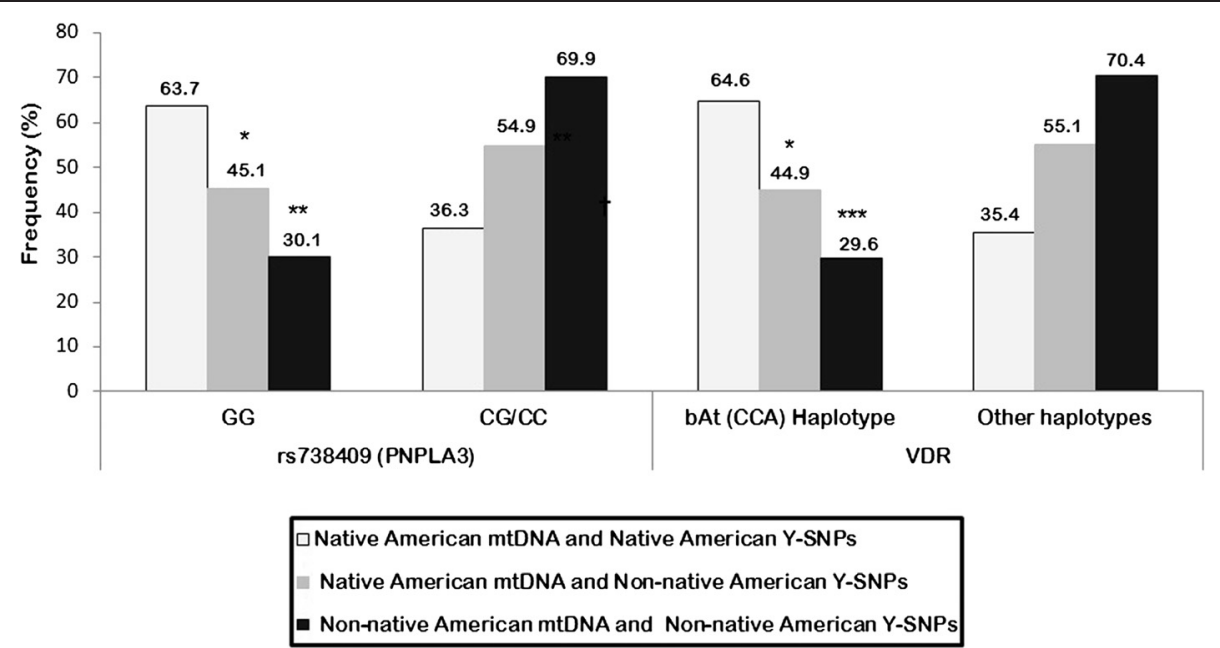

Fig. 4 Influence of maternal and paternal ethnic components on the genotype distribution. Prevalence of SNP rs738409 (PNPLA3 gene) and bAt (CCA) haplotype (VDR gene) among samples of maternal and paternal Native American ancestry, Native American maternal ancestry and Non-native American paternal ancestry (admixed samples) and Non-native American maternal and paternal ancestry. ${ }^{*} p=0.03$ when comparing samples with maternal and paternal Native American ancestry with the admixed group; ${ }^{* *} p=0.001$ and ${ }^{* * *} p=0.0008$ when comparing samples with maternal and paternal Native American ancestry with those exhibiting Non-native American maternal and paternal ancestry

European component. In addition, the process of admixture was -as in the rest of South America- sexbiased, generally involving a Native American maternal lineage and a Non-native American paternal lineage [32]. This finding is consistent with fact that the admixed group included in this study was characterized by Native American maternal ancestry and Nonnative American paternal haplogroups.

In general, men are 2-fold more likely to die from chronic liver disease and cirrhosis than are women. Liver transplant occurs less commonly in women than in men, with variable disease outcomes based on etiology [34]. A prospective study which analyzed epidemiological aspects of HCC in South America revealed that the situation in this region is no different from that in the rest of the world [35]. In this study, only male subjects were enrolled to assess both maternal and paternal lineages and determine the frequency of admixture in the studied population. In spite of this, the genotype prevalence of the studied SNPs must be considered as representative of the general population as it could not be modified by the lack of women in the recruited sample. However, it must be acknowledged that the interpretation of the results presented in this study may be affected by the possibility of sex difference in the genetic association of these polymorphisms and disease susceptibility. In fact, a negative correlation between male sex and the effect of rs738409 on liver fat content has been reported in NAFLD patients [36].

In this report, the prevalence of the genetic polymorphisms associated with risk of chronic liver disease in Non-native and Native American samples is in agreement with previously described frequencies in European and South American populations [18, 20, 37].

Regarding the GG genotype of SNP rs738409 in PNPLA3 gene, its frequency was significantly higher in individuals with Native American ancestry when compared with those exhibiting Non-native American haplogroups. In consistency with our results, a high prevalence of the unfavorable genotype was reported in Hispanics and in Argentinean patients with NALFD [18, 20]; but unfortunately, the ancestry components of these previously studied populations remain unknown.

Furthermore, the analysis of $V D R$ polymorphisms carried out in our study revealed intermediate frequencies between those reported for European and Asian samples, but similar to those previously published for Hispanics [37]. This result could be related to the high admixture levels observed in these populations, which could be explained by the combined ancestry of mestizos and the fact that Native American populations are descendants of nomadic Asian communities [37].

In different populations, haplotype structure analysis of $V D R$ gene has shown high genetic heterogeneity involving different haplotype blocks [37, 38]. In fact, studies carried out in admixed populations has revealed the importance of genetic heterogeneity since linkage disequilibrium increases or breaks down differently in different populations $[39,40]$. Considering the recent admixture of Latin American people, the variation in the patterns of linkage disequilibrium is not surprising in view of demographic events and genetic factors such as drift and recombination during the process of admixture. 
From the genetic perspective, Hispanics or Latinos generally represent an intricate mixture of European, Native American, and African lineages, with the proportion of each ancestry component typically depending on country or area of origin [41]. Recent studies that have examined the relationships between self-identified ethnicity, self-estimated admixture proportions and genetic marker estimated admixture proportions revealed that Hispanics underestimate their degree of Native American admixture, confirming that ethnic self-identification is likely to be complex due to the heterogeneity in individual admixture proportions and social environments within this group $[32,42]$. Due to its complex diversity, data obtained from relatively well-defined ethnic groups cannot be extrapolated to the majority of the South American population.

Interethnic admixture is either common or rapidly increasing in many -if not most- populations as a result of human migrations. Therefore, in agreement with our results, molecular evaluation of ancestry is strongly recommended in studies including this type of multi-ethnic populations [43]. From this point of view, the South American population, with Native American, European and African ancestral roots, and five centuries of vast interethnic admixing, offers a unique model for examining the impact of admixture on the conceptual development and clinical implementation of the results obtained from genomics medicine studies.

The results presented in this study intended to shed some light on the influence of ethnicity on the distribution of genetic polymorphisms associated with chronic liver disease in multi-ethnic and admixed populations. However, due to the multifactorial aspect of chronic liver diseases, the high prevalence of PNPLA3 and VDR genetic factors in Native Americans cannot be considered as the sole reason for the higher susceptibility of this ethnic group. Therefore, for the proper interpretation of the results presented herein, it must be taken into consideration that other genetic (e.g., SAMM50, $P A R V B, T M 6 S F 2$ ), environmental and behavioral factors may -as a whole- contribute to the higher risk of chronic liver disease in Native Americans [1-6, 16, 17]. In fact, the Hispanic population has high rates of obesity, diabetes and metabolic syndrome [44] which suggest that other nutritional and genetic factors exert a role in the increased risk for chronic liver disease in this population, as well. Unfortunately, the prevalence of chronic liver disease in subjects with Native American ethnic components in the studied area remains unknown to clarify this hypothesis.

In future decades, the prevalence of risk factors for chronic liver diseases, such as obesity, alcohol abuse and HCV chronic infection, is expected to rise $[1,2]$. Hence, the identification of effective genetic markers for chronic liver disorders would be priceless as it would allow us to stratify populations according to susceptibility [45]. As a consequence, in areas exhibiting a highly admixed genetic background with a significant contribution of Native American ancestry components -and, therefore, less favorable genetic variants-, the results presented in this study are of substantial relevance for the regional public health.

\section{Conclusion}

In conclusion, this study shows -for the first time- that more than $60 \%$ of individuals with Native American ancestry are carriers of high risk genetic polymorphisms related to chronic liver disease and progression towards liver cancer. These data not only support the molecular evaluation of ancestry in multi-ethnic population studies, but also suggest that the characterization of these variants among the healthy South American population may be useful for establishing public health policies aimed at the organization of awareness-raising campaigns for high risk ethnic communities.

\section{Methods}

\section{Study population}

During the period 2012-2013, a total of 258 unrelated male volunteers (mean age \pm standard deviation: 35.2 years \pm 11.5) were recruited by the DNA and blood bank at the Italian Hospital of Buenos Aires and the blood bank at the "Sardá" Maternity Hospital in Buenos Aires, Argentina. Both blood banks are located in different points of Buenos Aires city and provide attention to all strata of the general population (both Argentinean residents and South American immigrants), reinforcing sample's representativeness of the population of this region.

Male gender was considered as an inclusion criteria for participation in the study in order to determine both maternal (mitochondrial) and paternal (Y-chromosome) ancestry in all collected samples. Moreover, all enrolled subjects exhibited negative serology results for $\mathrm{HBV}$ and $\mathrm{HCV}$ infection (AxSYM, Abbott, Chicago, IL, USA) to avoid the dominant role that those viruses play on the development of chronic liver disease.

\section{Ethics, consent and permissions}

This study has been performed in accordance with the Declaration of Helsinki. Informed consent was obtained from each subject according to the experimental research protocol, approved by the Ethics Committee on Research from the Italian Hospital of Buenos Aires (CEPI N ${ }^{\circ} 1918$ ).

\section{Isolation of genomic DNA and genotype of PNPLA3 and VDR}

Genomic DNA was extracted from $1 \mathrm{ml}$ of EDTA blood by using FlexiGene ${ }^{\odot}$ DNA Kit (QIAGEN, GmbH, Hilden, Germany) following the manufacturer's protocol. 
Primers for SNPs rs738409 (PNPLA3) were designed by using Primer3 software [46]. A 668-bp fragment of PNPLA3 gene was amplified by the polymerase chain reaction $(\mathrm{PCR})$ technique using primers 5 '-CGA TCT AGC CCC TTT CAG TC-3' (forward) and 5'-GCA GAT TAA GTG AAC CAG CC-3' (reverse). PCR reaction was carried out for 30 cycles consisting of denaturation for $30 \mathrm{~s}$ at $94{ }^{\circ} \mathrm{C}$, annealing for $30 \mathrm{~s}$ at $62{ }^{\circ} \mathrm{C}$ and extension for $1 \mathrm{~min}$ at $72{ }^{\circ} \mathrm{C}$. With regard to SNPs for $V D R$ gene, the sets of primers used for amplification and their PCR cycling conditions were obtained from previously published studies $[47,48]$.

The PCR amplified fragments were bi-directionally sequenced using Big-Dye Termination chemistry system (Applied Biosystems, Life Technologies Corp., Foster City, CA, USA). In order to discriminate between homozygotes and heterozygotes, the sequencing chromatogram was examined by using BioEdit Sequence Alignment Editor version 7.1.3.0.

\section{Molecular evaluation of ancestry}

Haplogroups in mtDNA (haplogroups A2, B2, C and D1) and Y-SNPs (haplogroups E1b1b, G2a, I, J2, R1b1b2, Q1a3a) were assessed by Real-Time PCR followed by High Resolution Melting as previously described [49].

\section{Statistical analyses}

Statistical analyses were carried out using Fisher's exact test (group by group comparisons) and a value $p<0.05$ was considered as statistically significant. Furthermore, Holm-Bonferroni correction was used to address the problem of multiple hypothesis testing.

The correlation between the observed number of homozygous and heterozygous individuals and the numbers statistically expected from the Hardy-Weinberg equilibrium was assessed by the Chi-square goodness-of-fit test.

\section{Additional files}

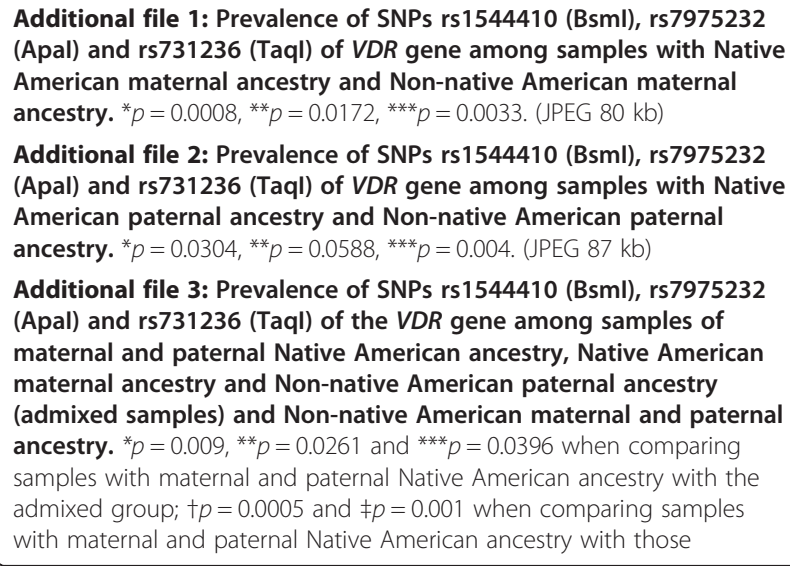

exhibiting Non-native American maternal and paternal ancestry. (JPEG $86 \mathrm{~kb})$

\section{Abbreviations}

EDTA: Ethylenediaminetetraacetic acid; HBV: Hepatitis B virus; HCC: Hepatocellular carcinoma; HCV: Hepatitis C virus;

mtDNA: Mitochondrial DNA; n: Sample size; NAFLD: Non-alcoholic fatty liver disease; NASH: Non-alcoholic steatohepatitis; PCR: Polymerase chain reaction; PNPLA3: Patatin-like phospholipase 3; SD: Standard deviation; SNP: Single-nucleotide polymorphism; VDR: Vitamin D receptor; Y-SNPs: Y-chromosome single-nucleotide polymorphisms.

\section{Competing interests}

The authors declare that they have no competing interests.

\section{Authors' contributions}

ACP molecular study, redaction of the manuscript, statistic study. JT analysis of data, redaction of the manuscript. MLH and MC contribution to molecular study. LF, LBP, AF, OT, FN, AG recruitment of samples and preparation of data. PA analysis of data. DC and DF conception of the study, analysis of data. All authors read and approved the final manuscript.

\section{Acknowledgements}

The authors are deeply grateful to the volunteers for their cooperation and also thank Tech. Noelia Bravo, Fiorella Caro, BSc and Ms Valeria Cabrera for technical assistance. This research was supported in part by grants from

"Instituto de Ciencias Básicas y Medicina Experimental" (ICBME) and

"Fundación para el Desarrollo de las Ciencias Básicas" (FUCIBA).

\section{Author details}

${ }^{1}$ Instituto de Ciencias Básicas y Medicina Experimental (ICBME), Hospital Italiano de Buenos Aires, Potosí 4240, C1199ACL Buenos Aires, Argentina. ${ }^{2}$ National Scientific and Technical Research Council (CONICET), Av. Rivadavia 1917, C1033AAJ Buenos Aires, Argentina. ${ }^{3}$ Servicio de Huellas Digitales Genéticas, Facultad de Farmacia y Bioquímica, Universidad de Buenos Aires, Junín 954, C1113AAD Buenos Aires, Argentina. ${ }^{4}$ Servicio de Medicina Transfusional, Hospital Italiano de Buenos Aires, Juan D. Perón 4190, C1181ACH Buenos Aires, Argentina. ${ }^{5}$ Servicio de Medicina Transfusional, Hospital Materno Infantil "Ramón Sardá", Esteban de Luca 2151, C1246ABQ Buenos Aires, Argentina. ${ }^{6}$ Servicio de Hepatología, Hospital Italiano de Buenos Aires, Juan D. Perón 4190, C1181ACH Buenos Aires, Argentina. ${ }^{7}$ Cátedra de Virología, Facultad de Farmacia y Bioquímica, Universidad de Buenos Aires, Junín 954, C1113AAD Buenos Aires, Argentina.

Received: 10 February 2015 Accepted: 17 July 2015

Published online: 29 July 2015

\section{References}

1. Bell BP, Manos MM, Zaman A, Terrault N, Thomas A, Navarro VJ, et al. The epidemiology of newly diagnosed chronic liver disease in gastroenterology practices in the United States: results from population-based surveillance. Am J Gastroenterol. 2008;103:2727-36.

2. Kim Y, Ejaz A, Tayal A, Spolverato G, Bridges JF, Anders RA, et al. Temporal trends in population-based death rates associated with chronic liver disease and liver cancer in the United States over the last 30 years. Cancer. 2014;120:3058-65.

3. Altekruse SF, McGlynn KA, Reichman ME. Hepatocellular carcinoma incidence, mortality, and survival trends in the United States from 1975 to 2005. J Clin Oncol. 2009;27:1485-91.

4. Mittal S, El-Serag HB. Epidemiology of hepatocellular carcinoma: consider the population. J Clin Gastroenterol. 2013;47(Suppl):S2-6.

5. Serfaty L, Lemoine M. Definition and natural history of metabolic steatosis: clinical aspects of NAFLD, NASH and cirrhosis. Diabetes Metab. 2008;34:634-7.

6. Vernon G, Baranova A, Younossi ZM. Systematic review: the epidemiology and natural history of non-alcoholic fatty liver disease and non-alcoholic steatohepatitis in adults. Aliment Pharmacol Ther. 2011;34:274-85.

7. Farrell GC, Larter CZ. Nonalcoholic fatty liver disease: from steatosis to cirrhosis. Hepatology. 2006;43(2 Suppl 1):S99-112. 
8. Ascha MS, Hanouneh IA, Lopez R, Tamimi TA, Feldstein AF, Zein NN. The incidence and risk factors of hepatocellular carcinoma in patients with nonalcoholic steatohepatitis. Hepatology. 2010;51:1972-8.

9. Nguyen GC, Thuluvath PJ. Racial disparity in liver disease: biological, cultural, or socioeconomic factors. Hepatology. 2008;47:1058-66.

10. Verma S, Bonacini M, Govindarajan S, Kanel G, Lindsay KL, Redeker A. More advanced hepatic fibrosis in hispanics with chronic hepatitis $C$ infection: role of patient demographics, hepatic necroinflammation, and steatosis. Am J Gastroenterol. 2006;101:1817-23.

11. Cheung RC, Currie S, Shen H, Ho SB, Bini EJ, Anand BS, et al. Chronic hepatitis $C$ in Latinos: natural history, treatment eligibility, acceptance, and outcomes. Am J Gastroenterol. 2005;100:2186-93.

12. Weston SR, Leyden W, Murphy R, Bass NM, Bell BP, Manos MM, et al. Racial and ethnic distribution of nonalcoholic fatty liver in persons with newly diagnosed chronic liver disease. Hepatology. 2005;41:372-9.

13. Dongiovanni $P$, Romeo $S$, Valenti L. Hepatocellular carcinoma in nonalcoholic fatty liver: role of environmental and genetic factors. World J Gastroenterol. 2014;20:12945-55.

14. Nahon P, Zucman-Rossi J. Single nucleotide polymorphisms and risk of hepatocellular carcinoma in cirrhosis. J Hepatol. 2012;57:663-74.

15. Sookoian S, Pirola CJ. The genetic epidemiology of nonalcoholic fatty liver disease: toward a personalized medicine. Clin Liver Dis. 2012;16:467-85.

16. Sookoian S, Castaño GO, Scian R, Mallardi P, Fernández Gianotti T, Burgueño $\mathrm{AL}$, et al. Genetic variation in transmembrane 6 superfamily member 2 and the risk of nonalcoholic fatty liver disease and histological disease severity. Hepatology. 2015;61:515-25.

17. Kitamoto $\mathrm{T}$, Kitamoto A, Yoneda M, Hyogo H, Ochi H, Nakamura T, et al. Genome-wide scan revealed that polymorphisms in the PNPLA3, SAMM50, and PARVB genes are associated with development and progression of nonalcoholic fatty liver disease in Japan. Hum Genet. 2013;132:783-92.

18. Romeo S, Kozlitina J, Xing C, Pertsemlidis A, Cox D, Pennacchio LA, et al. Genetic variation in PNPLA3 confers susceptibility to nonalcoholic fatty liver disease. Nat Genet. 2008;40:1461-5.

19. Dongiovanni $\mathrm{P}$, Donati $\mathrm{B}$, Fares $\mathrm{R}$, Lombardi R, Mancina RM, Romeo S, et al. PNPLA3 1148M polymorphism and progressive liver disease. World J Gastroenterol. 2013;19:6969-78.

20. Sookoian S, Castaño GO, Burgueño AL, Gianotti TF, Rosselli MS, Pirola CJ. A nonsynonymous gene variant in the adiponutrin gene is associated with nonalcoholic fatty liver disease severity. J Lipid Res. 2009;50:2111-6.

21. Trépo E, Pradat P, Potthoff A, Momozawa Y, Quertinmont E, Gustot T, et al. Impact of patatin-like phospholipase-3 (rs738409 C>G) polymorphism on fibrosis progression and steatosis in chronic hepatitis C. Hepatology. 2011;54:60-9.

22. Zhang L, You W, Zhang $H$, Peng $R$, Yao A, Li X, et al. PNPLA3 Polymorphisms (rs738409) and Non-Alcoholic Fatty Liver Disease Risk and Related Phenotypes: A Meta-Analysis. J Gastroenterol Hepatol. 2015; 16. doi:10.1111/jgh.12889.

23. Arteh J, Narra S, Nair S. Prevalence of vitamin D deficiency in chronic liver disease. Dig Dis Sci. 2010;55:2624-8

24. Kitson MT, Roberts SK. D-livering the message: The importance of vitamin D status in chronic liver disease. J Hepatol. 2012;57:897-909.

25. Targher G, Bertolini L, Scala L, Cigolini M, Zenari L, Falezza G, et al. Associations between serum 25-hydroxyvitamin D3 concentrations and liver histology in patients with non-alcoholic fatty liver disease. Nutr Metab Cardiovasc Dis. 2007;17:517-24

26. Dasarathy J, Periyalwar P, Allampati S, Bhinder V, Hawkins C, Brandt P, et al. Hypovitaminosis $D$ is associated with increased whole body fat mass and greater severity of non-alcoholic fatty liver disease. Liver Int. 2014;34:e118-27

27. Uitterlinden AG, Fang Y, Van Meurs JB, Pols HA, Van Leeuwen JP. Genetics and biology of vitamin D receptor polymorphisms. Gene. 2004;338:143-56.

28. Falleti E, Bitetto D, Fabris C, Cussigh A, Fontanini E, Fornasiere E, et al. Vitamin D receptor gene polymorphisms and hepatocellular carcinoma in alcoholic cirrhosis. World J Gastroenterol. 2010;16:3016-24.

29. Baur K, Mertens JC, Schmitt J, Iwata R, Stieger B, Eloranta JJ, et al. Combined effect of $25-\mathrm{OH}$ vitamin D plasma levels and genetic Vitamin D receptor (NR 111) variants on fibrosis progression rate in HCV patients. Liver Int. 2012:32:635-43.

30. Hung CH, Chiu YC, Hu TH, Chen CH, Lu SN, Huang CM, et al. Significance of vitamin d receptor gene polymorphisms for risk of hepatocellular carcinoma in chronic hepatitis C. Transl Oncol. 2014;7:503-7.
31. Catelli ML, Alvarez-Iglesias V, Gómez-Carballa A, Mosquera-Miguel A, Romanini C, Borosky A, et al. The impact of modern migrations on present-day multi-ethnic Argentina as recorded on the mitochondrial DNA genome. BMC Genet. 2011;12:77.

32. Corach D, Lao O, Bobillo C, van Der Gaag K, Zuniga S, Vermeulen M, et al. Inferring continental ancestry of argentineans from Autosomal, Y-chromosomal and mitochondrial DNA. Ann Hum Genet. 2010;74:65-76.

33. Flichman DM, Blejer JL, Livellara BI, Re VE, Bartoli S, Bustos JA, et al. Prevalence and trends of markers of hepatitis B virus, hepatitis $C$ virus and human Immunodeficiency virus in Argentine blood donors. BMC Infect Dis. 2014;14:218

34. Guy J, Peters MG. Liver disease in women: the influence of gender on epidemiology, natural history, and patient outcomes. Gastroenterol Hepatol (N Y). 2013;9:633-9.

35. Méndez-Sánchez N, Ridruejo E, Alves de Mattos A, Chávez-Tapia NC, Zapata R, Paraná $R$, et al. Latin American Association for the Study of the Liver (LAASL) clinical practice guidelines: management of hepatocellular carcinoma. Ann Hepatol. 2014;13 Suppl 1:4-40.

36. Sookoian S, Pirola CJ. Meta-analysis of the influence of I148M variant of patatin-like phospholipase domain containing 3 gene (PNPLA3) on the susceptibility and histological severity of nonalcoholic fatty liver disease. Hepatology. 2011;53:1883-94

37. Ingles SA, Haile RW, Henderson BE, Kolonel LN, Nakaichi G, Shi CY, et al. Strength of linkage disequilibrium between two vitamin $D$ receptor markers in five ethnic groups: implications for association studies. Cancer Epidemiol Biomarkers Prev. 1997;6:93-8.

38. Lins TC, Vieira RG, Grattapaglia D, Pereira RW. Population analysis of vitamin $D$ receptor polymorphisms and the role of genetic ancestry in an admixed population. Genet Mol Biol. 2011;34:377-85.

39. Liu N, Sawyer SL, Mukherjee N, Pakstis AJ, Kidd JR, Kidd KK, et al. Haplotype block structures show significant variation among populations. Genet Epidemiol. 2004;27:385-400.

40. Sawyer SL, Mukherjee N, Pakstis AJ, Feuk L, Kidd JR, Brookes AJ, et al. Linkage disequilibrium patterns vary substantially among populations. Eur J Hum Genet. 2005;13:677-86.

41. Klimentidis YC, Miller GF, Shriver MD. Genetic admixture, self-reported ethnicity, self-estimated admixture, and skin pigmentation among Hispanics and Native Americans. Am J Phys Anthropol. 2009;138:375-83.

42. Tang H, Quertermous T, Rodriguez B, Kardia SL, Zhu X, Brown A, et al. Genetic structure, self-identified race/ethnicity, and confounding in case-control association studies. Am J Hum Genet. 2005;76:268-75.

43. Trinks J, Hulaniuk ML, Caputo M, Pratx LB, Ré V, Fortuny L, et al. Distribution of genetic polymorphisms associated with hepatitis $\mathrm{C}$ virus $(\mathrm{HCV})$ antiviral response in a multiethnic and admixed population. Pharmacogenomics J. 2014;14:549-54.

44. Li Q, Qu HQ, Rentfro AR, Grove ML, Mirza S, Lu Y, et al. PNPLA3 polymorphisms and liver aminotransferase levels in a Mexican American population. Clin Invest Med. 2012;35:237-45.

45. Hong $\mathrm{H}$, Tong W. Emerging efforts for discovering new biomarkers of liver disease and hepatotoxicity. Biomark Med. 2014;8:143-6.

46. Rozen S, Skaletsky H. Primer3 on the WWW for general users and for biologist programmers. Methods Mol Biol. 2000;132:365-86.

47. Quesada JM, Casado A, Díaz C, Barrios L, Cuenca-Acevedo R, Dorado G. Allele-frequency determination of Bsml and Fokl polymorphisms of the VDR gene by quantitative real-time PCR (QRT-PCR) in pooled genomic DNA samples. J Steroid Biochem Mol Biol. 2004;89-90:209-14.

48. Gunes S, Bilen CY, Kara N, Asci R, Bagci H, Yilmaz AF. Vitamin D receptor gene polymorphisms in patients with urolithiasis. Urol Res. 2006:34:47-52.

49. Zuccarelli G, Alechine E, Caputo M, Bobillo C, Corach D, Sala A. Rapid screening for Native American mitochondrial and Y-chromosome haplogroups detection in routine DNA analysis. Forensic Sci Int Genet. 2011;5:105-8. 